\title{
HIV Pre-Exposure Prophylaxis Adherence Test Using Reverse Transcription Isothermal Amplification Inhibition Assay
}

Jane Zhang

University of Washington

Yu Zhang

University of Washington

Benjamin Sullivan

University of Washington

Ayokunle Olanrewaju

University of Washington

Andrew Bender

University of Washington

Lorraine Lillis

PATH

David Boyle

PATH

Paul Drain

University of Washington

Jonathan Posner ( $\boldsymbol{\nabla}$ jposner@uw.edu )

University of Washington

\section{Research Article}

Keywords: ART, PrEP, NRTI, RPA

Posted Date: December 3rd, 2020

DOl: https://doi.org/10.21203/rs.3.rs-113196/v1

License: (c) (i) This work is licensed under a Creative Commons Attribution 4.0 International License.

Read Full License

Version of Record: A version of this preprint was published at Analytical Methods on January 1st, 2022. See the published version at https://doi.org/10.1039/D2AY00008C. 



\title{
HIV Pre-Exposure Prophylaxis Adherence Test using Reverse Transcription Isothermal Amplification Inhibition Assay
}

\author{
Jane Y. Zhang ${ }^{1}$, Yu Zhang ${ }^{2}$, Benjamin P. Sullivan ${ }^{1}$, Ayokunle O. Olanrewaju ${ }^{1}$, Andrew T. Bender ${ }^{1}$, Lorraine \\ Lillis $^{3}$, David Boyle ${ }^{3}$, Paul K. Drain ${ }^{4,5,6}$, Jonathan D. Posner (corresponding author, jposner@uw.edu) ${ }^{1,2,5}$ \\ ${ }^{1}$ Department of Mechanical Engineering, University of Washington \\ ${ }^{2}$ Department of Chemical Engineering, University of Washington \\ ${ }^{3} \mathrm{PATH}$ \\ ${ }^{4}$ Department of Global Health, University of Washington \\ ${ }^{5}$ Department of Medicine, University of Washington \\ ${ }^{6}$ Department of Epidemiology, University of Washington
}

\begin{abstract}
:
Current HIV antiretroviral (ART) or pre-exposure prophylaxis (PrEP) therapy adherence monitoring relies on either patient self-reported adherence or monitoring drug dispensing, which are not reliable. We propose an objective adherence monitoring assay which directly measures nucleotide reverse transcriptase inhibitor (NRTI) concentration using a reverse transcription isothermal amplification inhibition assay. We measure the concentration of Tenofovir diphosphate (TFV-DP), a deoxyadenosine triphosphate (dATP) analog and an active NRTI compound and long-term adherence marker for PrEP, by measuring the inhibition of the reverse transcription of an RNA template. The completion or inhibition of reverse transcription is evaluated by Recombinase Polymerase Amplification (RPA), an isothermal nucleic acid amplification assay. We present and validate a model that predicts the amplification probability as a function of dATP and TFV-DP concentrations, nucleotide binding sites on the RNA template, and the RNA template concentration. The model helps to rationally design and optimize the assay to operate at clinically relevant TFV-DP concentrations. We provide statistical analysis that demonstrates how the assay can be used as a qualitative or semi-quantitative tool for measuring adherence to NRTI drugs and used to support patient compliance.
\end{abstract}




\section{Introduction}

The number of people living with HIV (PLWH) is approximately 38 million globally, ${ }^{1}$ including 1.1 million in the US. ${ }^{2}$ To reduce new incidences, the WHO recommends pre-exposure prophylaxis (PrEP) to all people at risk of HIV infection. ${ }^{3}$ When taken daily, PrEP reduces the risk of contracting HIV by more than $90 \% .^{4}$ In the US, 770,000 are prescribed PrEP and 1.2 million people are considered high risk for HIV and indicated for PrEP. ${ }^{5}$

Despite increasing number of people receiving ART and PrEP, adherence to the therapies is still challenging for various environmental, social, economic, and behavioral reasons. ${ }^{2,6,7,8,9}$ Given the strong correlation of adherence to ART and PrEP with treatment success, ${ }^{8,10}$ continuous efforts and improvement along the patient care continuum are aimed at timely adherence monitoring and support. ${ }^{11,12}$

Current methods for monitoring adherence rely primarily on patient self-reporting. ${ }^{11}$ Self-reporting adherence is inexpensive to implement; however, it has many known limitations such as memory lapse and social desirability bias which undermine its value. ${ }^{13-15}$ Many device-based adherence monitoring approaches such as digital pillbox, ${ }^{16,17}$ Micro Electro Mechanical System-functionalized pills, ${ }^{18}$ textmessage and electronic medication reminders ${ }^{19-22}$ can be either too costly or do not provide proof of pill ingestion, thus hindering their adoption. Direct drug level quantification based on patient sample such as in blood or urine can provide objective, accurate, and unbiased measurement directly reflective of adherence. . $^{13,14,23}$

Tenofovir disoproxil fumarate (TDF) is the prodrug in all PrEP combination regimens currently recommended by the WHO and approved by the US Food and Drug Administration (FDA), and a principal component of first line ART regimens. ${ }^{24,25,26,27,28}$ Tenofovir is in a class of HIV therapy called nucleotide reverse transcriptase inhibitors (NRTIs), which act as nucleotide analogs to block reverse transcription (RT) of the HIV virus so that they cannot replicate inside the human cell. ${ }^{29,30}$ TFV has a short half-life $(\sim 15 \mathrm{hrs})$ in plasma ${ }^{31,32}$ and can be measured using liquid chromatography-mass spectroscopy (LCMS). ${ }^{33,34}$ LC-MS measurements require significant infrastructure, technical expertise, and investment, and is inconsistent with integration in the most clinic settings. ${ }^{28}$ There are also lateral flow point-of-care (POC) TFV competitive immunoassay in urine and blood. ${ }^{37-40}$ The assays can accurately classify recent dosage up to a week ${ }^{41}$ and have been used to identify poor adherence against LC-MS results. ${ }^{40}$ 
Unfortunately, because TFV has a relatively short half-life, the immunoassay results are susceptible to the white coat effect. ${ }^{39,42}$

Tenofovir-diphosphate (TFV-DP) is the active form of TFV that accumulates in the red blood cells with an intracellular half-life up to 17 days. ${ }^{32,43}$ As a result, TFV-DP level is a valuable indicator of long-term adherence and predictive of health outcomes. ${ }^{44,45,46,33}$ The median concentration of TFV-DP in red blood cells, as measured by LC-MS, ranges from 15 to $170 \mathrm{fmol} / 10^{6}$ red blood cells (RBCs) from 1 dose per week to 7 doses per week. ${ }^{32}$ With average hematocrit of $40 \%$ and an average RBC count of $5 \times 10^{6}$ $\mathrm{RBCs} / \mu \mathrm{L}$ for adults, 15 to $170 \mathrm{fmol} / 10^{6}$ red blood cells translates to a clinical range of $75 \mathrm{nM}$ to $850 \mathrm{nM}$ of TFV-DP in red blood cells. ${ }^{34}$ A study that correlated LC-MS TFV-DP concentrations from dry blood spots against HIV contraction outcomes showed that PrEP clients taking $\geq 4$ doses/week were protected from HIV infection. ${ }^{45,47}$ Four doses per week as measured by LC-MS was $700 \mathrm{fmol} / \mathrm{punch}^{47}$ in a dried blood spot or $292 \mathrm{nM}$ in red blood cells and has been suggested as an adherence cutoff threshold for distinguishing adherence versus non-adherence. Recently, our group reported on an enzymatic assay, termed REverSe TRanscrIptase Chain Termination (RESTRICT), that measures DNA duplex production by reverse transcriptase in the presence of NRTI drugs. ${ }^{48,49}$ The assay accurately distinguished TFV-DP drug levels within the clinical range for adherence and has the potential to be a useful test to identify patients with poor adherence to ART and PrEP. ${ }^{48}$ Real-time PCR has also been used to quantify inhibitory concentrations of NRTIs in drug development against HIV-1 RT. ${ }^{50,51,52}$ PCR or other isothermal amplification methods have not been used to measure the presence of TFV-DP.

In this paper, we report on the development on an assay to measure TFV-DP concentration using reverse transcription (RT) followed by Recombinase Polymerase Amplification (RPA). RPA is an enzyme-driven isothermal nucleic acid amplification method, often used as an infectious disease diagnostic in low resource settings. ${ }^{58,54,55}$ The concentration of TFV-DP is inferred from the inhibition of the reserve transcription of a RNA template by chain termination of the reverse transcription of a template RNA by a TFV-DP. If the template is not transcribed by RT because the process has been inhibited by the presence of a NRTI, RPA cannot amplify the template. The assay is probabilistic and may require multiple runs on a single sample to determine the experimental probability. We present experimental results for measuring the TFV-DP concentration in the clinically relevant range for ART and PrEP. The assay can be completed in under an hour and requires a single controlled temperature of $39{ }^{\circ} \mathrm{C}$ for both RT and RPA steps. We present an experimentally validated, analytical model that predicts the amplification probability as a 
function of dATP concentration, RNA template number, and number of inhibitive binding sites on the RNA target sequence. We show that the assay can be designed to provide a qualitative test tuned to a specific cut-off value, depending on the desired clinical information.

\section{Results}

\section{Theoretical Model}

The assay works by measuring the experimental probability that RNA templates are reverse transcribed by RT into cDNA that is subsequently amplified by RPA as shown in Figure 1. When no TFV-DP are present, we expect the RNA template to be reverse transcribed into complete cDNA. cDNA can then be amplified by RPA to provide a positive result. When a TFV-DP molecule is present, and is incorporated as a dATP analog during cDNA synthesis, chain termination occurs, resulting in partial length cDNA. If all, or nearly all, cDNAs are chain terminated, then there is insufficient cDNA template for RPA amplification. For intermediate concentrations, some samples have sufficient cDNA synthesized for amplification, while others do not, resulting in a probabilistic response.

Here we present an analytical model to predict the amplification probability as a function of the TFV-DP and dATP concentration, RNA template copy number, and number of inhibitive binding sites on the RNA target sequence. This model assumes there is sufficient RT enzyme, time, and temperature needed for reverse transcription to occur to completion and that dATPs or TFV-DPs are present at sufficient concentration that they are not consumed during the reaction. The model also assumes all cDNA synthesized from the RT reaction are amplified, therefore, the probability of amplification is equal to the probability of cDNA synthesis. The probability of reverse transcription or amplification depends on the concentrations of dATP, [dATP], and TFV-DP, [TFV-DP], expressed as the ratio, $R$, number of complementary binding sites to the TFV-DP on the RNA template, $s$, and the number of template molecules in the reaction mix, $t$. The relationship between $R, s, t$ and the modulated RPA readout in the analytical model is illustrated in Figure 1B.

For each complementary binding site on a template RNA molecule, the probability that dATP is incorporated by the RT is, 
$P_{\text {site }}=\frac{[d A T P]}{[d A T P]+K_{a f f} \cdot[T F V-D P]}=\frac{\frac{[d A T P]}{[T F V-D P]}}{\frac{[d A T P]}{[T F V-D P]}+K_{a f f} \cdot \frac{[T F V-D P]}{[T F V-D P]}}=\frac{R}{R+K_{a f f}}$,

where $K_{a f f}$ is the relative affinity of RT for a TFV-DP compared to its native dATP substrate and is determined empirically. Assuming that each binding event onto the complementary site on the RNA template is independent, and that there are a total number of $s$ binding sites, the probability for at least one TFV-DP molecule to bind to a single template RNA within the target sequence is given as,

$$
P_{\text {template }}=1-\left(P_{\text {site }}\right)^{S}=1-\left(\frac{R}{R+K_{\text {aff }}}\right)^{S}
$$

When a TFV-DP binds to the RNA template the chain is terminated, the synthesis of cDNA is halted, resulting in a partial-length complementary strand that will not be amplified in the subsequent RPA reaction. At least several, if not tens of copies, of cDNA must be synthesized during the RT step in order to amplify and obtain an RPA signal. If there are $t$ copies of the RNA template, and all of them need to be inhibited for RPA to be negative, the probability for amplification can be written as,

$P_{a m p}=1-\left[P_{\text {template }}\right]^{t}=1-\left[\left(1-\left(\frac{R}{R+K_{a f f}}\right)^{s}\right]^{t}\right.$

This simple analytical model was adopted for the inhibition of RT activity by TFV-DP in competition with dATP.

\section{Experimental Results}

The assay measures the probability that TFV-DP is inhibiting reverse transcription and subsequent isothermal amplification. We calibrated the assay to the appropriate clinical range of TFV-DP in ART and PrEP patients with the goal of using the assay to measure patient adherence to the drug treatment. The transition between no inhibition (low TFV-DP) and complete inhibition (high TFV-DP) occurs within a relatively narrow range of clinically relevant TFV-DP concentrations (between $75 \mathrm{nM}$ and $850 \mathrm{nM}$ in red blood cells).

First, we examined whether the RNA template incubated with RT and varying concentrations of TFV-DP will synthesize cDNA and enable subsequent amplification by RPA. Figure 2 shows baselined-adjusted 
and normalized RPA fluorescence readout as a function of time from RPA for 0, 100, 300, 600, and 1,250 nM TFV-DP spiked in the RT mix during the reverse transcription step. When TFV-DP content was low $(0$ and $100 \mathrm{nM}), 5$ out of 5 samples amplified, indicating that the RNA templates were successfully reverse transcribed into cDNA, as shown in Figure 2A. For moderate TFV-DP levels (300 nM), shown in Figure $2 \mathrm{C}$, only 5 out of 10 of the samples amplified, suggesting that in half of the samples, insufficient RNA templates were transcribed because of the chain-termination of TFV-DP. At higher concentrations of TFV-DP (600 and $1250 \mathrm{nM}$ ), only 1 out of 14 and 0 out of 5 amplified, respectively, indicating that nearly all RNA templates were chain-terminated by TFV-DP during the RT step, as shown in Figures 2D and 2E.

The experimental probability of amplification is plotted against the model in Figure 3 as a function of the TFV-DP concentration. Here, both the experiments and model have $7.8 \times 10^{3}$ copies of RNA template with 21 Uracil sites and $1.5 \mu \mathrm{M}$ dNTP. We fit $K_{a f f}=0.7$, which is similar to the values found previously. ${ }^{48,56}$ The experimental data and model show that the probability of amplification is unity when no TFV-DP is present, decreases rapidly with incremental addition of TFV-DP, and remains at zero when the TFV-DP concentration is high. Here the assay has been tuned to demonstrate sensitivity between $300 \mathrm{nM}$ and 500 $\mathrm{nM}$ of TFV-DP, within the shaded area between $75 \mathrm{nM}$ and $850 \mathrm{nM}$, which is the clinically relevant TFVDP concentration range for patients taking ART and PrEP. ${ }^{34,43}$

The sensitive range of the assay could be further shifted for different TFV-DP concentrations by varying $t$ or $s$. In Figure 4, analytical model outputs of the probability of amplification are plotted against $R$. $P_{a m p}$ shifts towards higher NRTI concentrations (left) as RNA template copy number, $t$, increases or the number of binding sites, $s$, decreases, respectively. With increasing amount of RNA template, $t$, the reaction is more likely to amplify because there are more chances to complete the reverse transcription, thus requiring more TFV-DP to inhibit the RT step. When there are increasing number of Uracil sites, $s$, on the template, it is more likely for a TFV-DP molecule to bind to one of the sites, thus inhibiting subsequent amplification. Each of the probability curves has a 50\% inhibition concentration (IC50) value where 50\% of the total reactions are inhibited. When $s$ increases or $t$ decreases, the probability curves shift right towards lower IC50 values (in terms of NRTI concentration) and smaller slope, and thus extend over a wider range of concentrations. Having a lower slope may be advantageous when the goal is to quantitatively measure drug levels. If $s$ decreases or $t$ increases, the probability curves shift left towards higher IC50 values (in terms of NRTI concentrations), and the curve becomes steeper. Steep probability 
curves are advantageous for qualitative tests where one wants to distinguish concentrations that are either above or below a certain threshold.

TFV-DP concentration can be deduced quantitatively from outcomes of repeated trials with the same experimental conditions using binomial probability and respective confidence intervals. Each reaction has a binomial outcome with an expected probability of amplification that can be derived from the analytical model. Multiple reactions can be performed to sample the expected probability. Given total number of trials of the same reaction on one sample $N$, and the number of amplified (positive) reactions $n$, the positive outcome over total number of repeated trials $C$ is defined as $C=n / N$, a binomial random variable with an expected mean of $P_{a m p}$ and confidence interval (C.I.) as estimated using the Clopper-Pearson method. The probability for $C$ can be expressed in terms of $[T F V-D P]$, such that for $95 \%$ of the reactions run, $C$ falls within the range $P_{a m p} \pm C$.I.. In Figure 5, the estimated $[T F V-D P]$ is plotted against $C$ for a specific $N$ (5 or 20 total reactions), $[d A T P]=0.375 \mu \mathrm{M}, s=21$ Uracil sites per template, and $t=7.8 \times 10^{3}$ copies of RNA template. When $\mathrm{N}=5$, we can see that for a given measured $\mathrm{C}$, the C.I. is large and it is difficult to measure the precise concentration of TFV-DP. This is especially true at $\mathrm{C}=1$ or $\mathrm{C}=0$ where the C.I. is asymmetric. For example, at $\mathrm{C}=0$, the lower bound is approximately $300 \mathrm{nM}$ and the upper limit is unbounded. When we increase the number of trials, shown in Figure $5 \mathrm{~b}$ for $\mathrm{N}=20$, the greater the number of repeated reactions narrows the C.I., indicating increasing confidence in the deduced TFV-DP concentrations. In addition, the deduced $[T F V-D P]$ has the tightest C.I. for intermediate values of $C$, and grows wider when $C$ approaches the minimum or maximum values of 0 (all repeated trials inhibited) or 1 (all repeated trials amplified), representing greater quantitative sensitivity obtained towards the intermediate $C$.

Alternatively, the assay could be used for qualitative measurement of TFV-DP concentration based on the outcome of a limited number of repeated trials, a one-tailed hypothesis, and $p$-values from hypothesis testing. Using a standard binomial distribution, with each $n, N$, and $P_{a m p}$, given mean $\mu=n P_{a m p}$, and variance $\sigma=\mathrm{nP}_{\mathrm{amp}}\left(1-\mathrm{P}_{\mathrm{amp}}\right)$, the $p$-value can be calculated based on the cumulative distribution function.

Table 1 shows a lookup table of $p$-values for the one-tailed hypothesis test that $[T F V-D P]>292 \mathrm{nM}$, as a function of the total number of trials, $N$, and the number of positive amplification reactions, $n$. This hypothesis test is clinically relevant because direct observation clinical studies show that TFV-DP greater than $292 \mathrm{nM}$, or four doses per week, is a good measure of adherence and represents protection from 
contracting HIV. ${ }^{47}$ If a patient's sample fails this hypothesis test, it suggests that the patient does not have sufficient TFV-DP concentration, they may have significant risk of contracting HIV, and should consider adherence counseling and support to improve adherence.

\section{Discussion}

The described method is the first time that an isothermal amplification has been applied for HIV antiretroviral adherence test. Isothermal amplification techniques are increasingly used for point of care diagnostics because they are sensitive, rapid, and have simple instrumentation requirements, making them suitable for deployment in the field or clinic-based settings. The method could be applied to measure other

NRTIs (e.g. Emtricitabine) to provide additional information on adherence for patients receiving combination therapies. One limitation is that RNA templates are relatively unstable and should be handled with care as repeated freeze-thawing would result in deteriorating of template copies. Further optimization of the experimental conditions, length, and costs could be performed by varying the input RT enzyme concentration, time for RT, or temperature. Further improvement in the sensitivity of the assay can be achieved with custom primer design to increase the length of RNA template during RT. Varying dNTP concentration could lead to tunability of the sensitivity range, making it an ideal test to identify suboptimal adherence in samples other than whole blood. Multiplexing of measurement with more than one dNTP concentration could lead to additional experimental data points and more accurate TFV-DP estimates. In addition, more quantitative signal intensity measurements from RPA may be obtained by including an internal amplification standard.

\section{Methods}

The assay process requires three steps: reverse transcription of the RNA template into cDNA, deactivation of RT enzyme, and RPA, as shown in Figure 1A. The entire process takes about 35 minutes from the beginning to finish, with only one controlled heating temperature of $39{ }^{\circ} \mathrm{C}$ in addition to an enzyme deactivation step at any temperature above $70{ }^{\circ} \mathrm{C}$.

Preparation of RNA template 
The RNA template used in this study was extracted from HIV virus (EQUAPOL, Duke University Medical Center, virus name DE00110CN001.S1 from cell culture supernatant). The RNA was extracted by following standard procedures with a spin-column (QIAamp Viral RNA Mini Kit, Cat No./ID: 52904, Valencia, CA). All RNA extracts were stored at $-20{ }^{\circ} \mathrm{C}$ until use. Quantification of the RNA was performed with real-time RT-PCR methods as previously published. ${ }^{57}$ To avoid unintended amplification by pro-viral DNA contaminants, we added DNase I (AMPD1-1KT, Sigma-Aldrich) to degrade DNA. The RNA samples were incubated with DNase for 15 minutes at room temperature, followed by 10 minutes at $70^{\circ} \mathrm{C}$.

\section{The RT assay}

The first step in the assay is reverse transcription of the RNA template into cDNA. cDNA synthesis was completed with final concentrations of 1x AffinityScript RT buffer (600107, Agilent Technologies, Santa

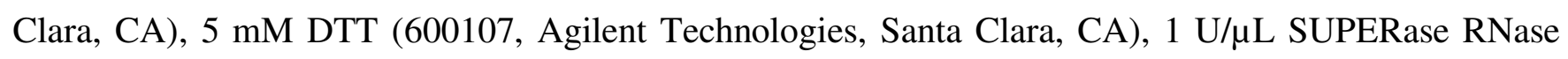
Inhibitor (AM2694, Invitrogen, Carlsbad, CA), 0.05 U/ $\mu$ L HIV Reverse Transcriptase (HIV RT P66/51, AbCam), $1.5 \mu \mathrm{M}$ or $3 \mu \mathrm{M}$ dNTP mix (R0192, Fisher Scientific, Houston, TX), and $0.42 \mu \mathrm{M}$ HIV pol reverse primer (Integrated DNA Technologies, Coralville, IA, USA) that contains the complementary sequence adopted from the proviral HIV-1 DNA pol gene. ${ }^{55}$ All reagents were diluted in nuclease free water (P1193, Promega, Madison WI). $7.8 \times 10^{3}$ copies of RNA templates and TFV-DP ranging from 0 and $1250 \mathrm{nM}$ (166403-66-3, BOC Sciences Inc.) were spiked in each reaction mix. The final reaction volume was $20 \mu \mathrm{L}$. The cDNA synthesis was performed at $39^{\circ} \mathrm{C}$ (T16, TwistDx Ltd., United Kingdom) for 10 minutes. After cDNA synthesis, RT enzyme was deactivated at $70{ }^{\circ} \mathrm{C}$ for 5 minutes in a dry bath to prevent further reverse transcription of RNA templates in the RPA reaction that contains much higher dNTP concentrations.

\section{The RPA assay}

After cDNA synthesis in the presence of TFV-DP, we amplified using RPA (TwistAmp® exo, TAEXO02KIT, Cambridge, United Kingdom) in order to detect the cDNA products that may or may not be present in the sample. ${ }^{58}$ Rehydration buffer from the kit was added to the lyophilized RPA reagent, reverse primers, forward primers, and probes ${ }^{55}$ were added for final respective concentrations of $0.54 \mu \mathrm{M}$, $0.54 \mu \mathrm{M}$, and $0.12 \mu \mathrm{M}$. 0.1\% BSA (AM2616, UltraPure, Fisher Scientific, Houston, TX) was added to 
enhance amplification yield. Nuclease free water (P1193, Promega, Madison WI) was added for a final volume of $37.5 \mu \mathrm{L}$ per reaction.

After RT deactivation, cDNA products were stored on ice. $10 \mu \mathrm{L}$ from each reaction was added to the $37.5 \mu \mathrm{L}$ RPA mix, and $2.5 \mu \mathrm{L}$ of $\operatorname{Mg}(\mathrm{Ac}) 2$ (TwistAmp® exo, TAEXO02KIT, Cambridge, United Kingdom) was added last to activate the RPA reactions with a total reaction volume of $50 \mu \mathrm{L}$. Preincubation was performed at $39^{\circ} \mathrm{C}$ for $5 \mathrm{~min}$, followed by a brief manual mixing. The mixed samples were incubated at $39{ }^{\circ} \mathrm{C}$ for an additional 20 minutes. Real-time detection of RPA via 6carboxyfluorescein (FAM) fluorescence was measured (T16, TwistDx Ltd., United Kingdom) every 20 seconds.

All fluorescence data were normalized between 0 and 1 with a baseline measurement at 360 seconds (after mixing was performed) and maximum fluorescence at 1,050 seconds from the positive control (no TFVDP added). Any increase in normalized fluorescence $(\Delta \mathrm{AU})$ greater than $10 \mathrm{AU}$ were considered positive. These same reactions were repeated for at least $n=5$ times to validate the sensitivity of the assay as hypothesized, and more than 5 time, at 10 and 14 times for TFV-DP concentration of $300 \mathrm{nM}$ and $600 \mathrm{nM}$ for additional confidence. We used different values of $n$ because of the different predicted probabilities and the statistical requirements to obtain appropriate confidence levels. The counts of positive and negative reactions were tallied to calculate the amplification probability.

\section{Acknowledgement}

We are grateful for funding from the NIH (R01AI136648, R21AI127200, R01EB022630). This work was conducted using equipment in the Biochemical Diagnostics Foundry for Translational Research supported by the M.J Murdock Charitable Trust. We are also grateful for helpful conversations with Dr. Rebecca Sandlin and Dr. Mehmet Toner at Harvard Medical School. Research reported in this publication was supported by the University of Washington / Fred Hutch Center for AIDS Research, an NIH-funded program under award number AI027757 which is supported by the following NIH Institutes and Centers: NIAID, NCI, NIMH, NIDA, NICHD, NHLBI, NIA, NIGMS, NIDDK. Figure 1 was created with BioRender.com. 


\section{Author contributions}

J.Y.Z. and Y.Z. performed all experiments. J.Y.Z, Y.Z., B.P.S. and A.O.O. designed the theoretical model. A.T.B. and L.L. suggested critical steps with the RT and RPA assay. D.B., P.K.D., and J.D.P. reviewed the manuscript and provided valuable feedback.

\section{Additional Information}

The author(s) declare no competing interests. 


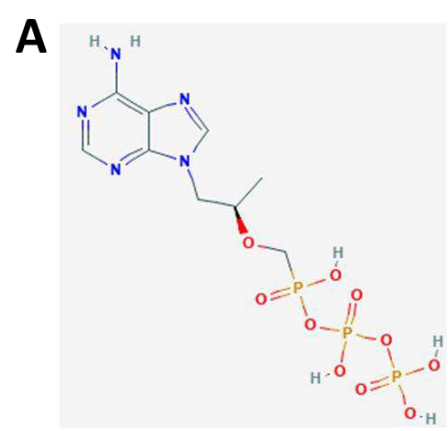

Tenofovir-Diphosphate

(TFV-DP)

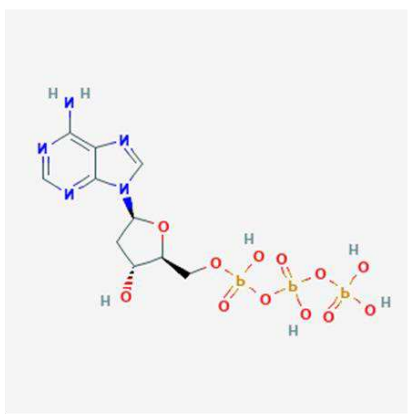

deoxyadenosine-Triphosphate (dATP)
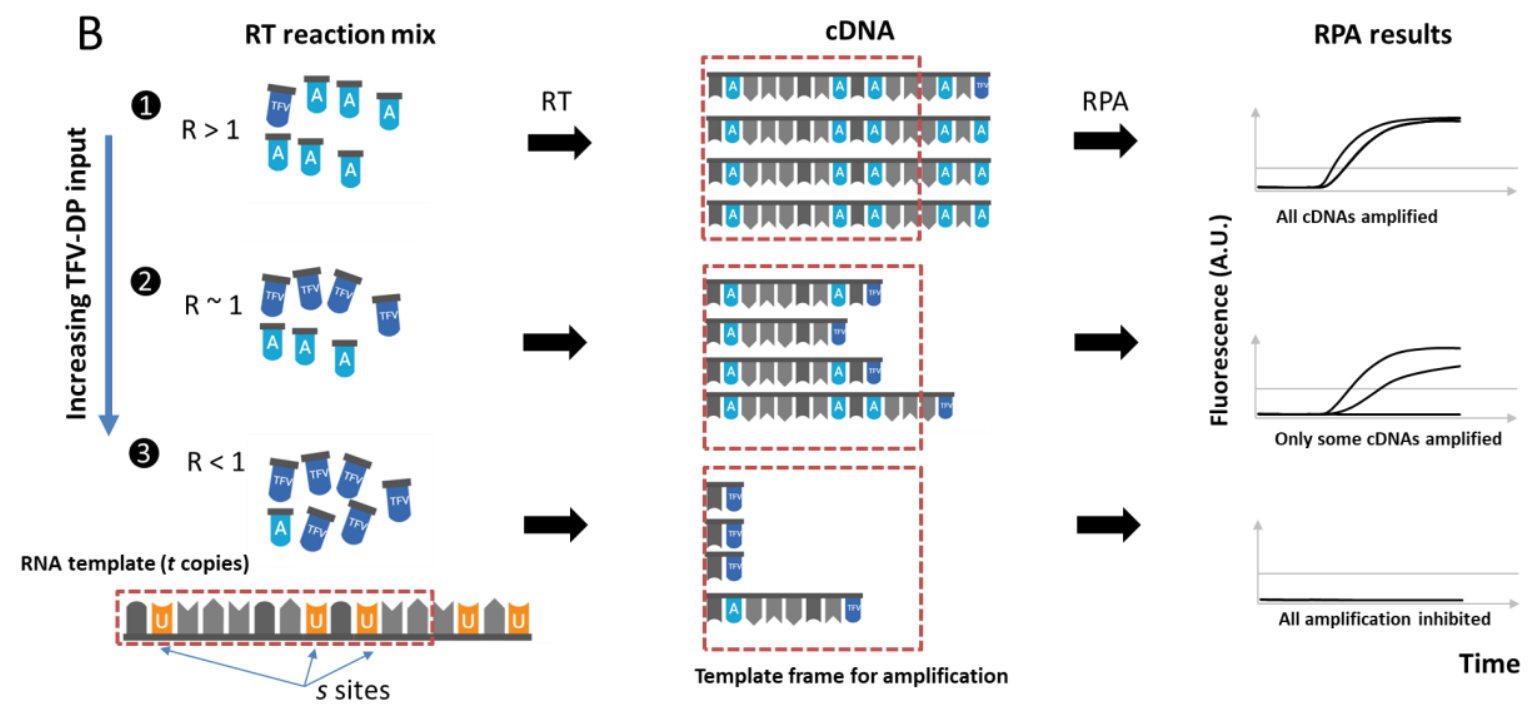

Figure 1. Overview of TFV-DP measurement using Reverse Transcription Isothermal Amplification Inhibition. (A) Molecular structural similarity between TFV-DP and dATP indicates that TFV-DP is a NRTI, blocking dATP binding to terminate HIV RT activities. ${ }^{59,60}$ (B). Modulation of the assay by competitive binding between dATP and TFV-DP during cDNA synthesis. The level of TFV-DP is determined by amplifying the RNA template that is reverse transcribed by RT. If there is little or no TFV-DP present, the RT reverse-transcribes the template RNA to form cDNA with a complete template frame for amplification, and the cDNA is amplified by RPA. (2) If there are moderate concentrations of TFV-DP compared to dATP, some of the cDNA chain may be terminated and thus only some full-length cDNA is formed. In this case only a fraction of amplification reactions will be positive. (3) When high concentrations of TFV-DP are present, nearly all cDNA is chain-terminated, resulting in RPA inhibition of all the samples. 

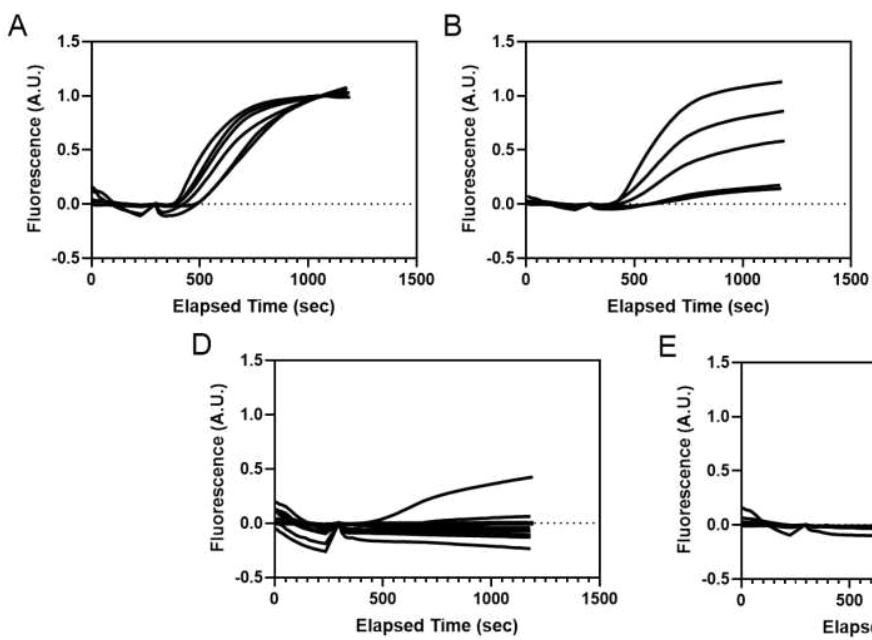

E
C

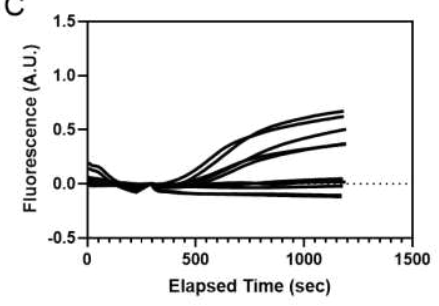



Figure 2. Real-time RPA fluorescence as a result of A) 0, B) $100 \mathrm{nM}, \mathrm{C}) 300 \mathrm{nM}$, D) $600 \mathrm{nM}$, and E) 1,250 nM TFV-DP spiked into the RT reactions with $1.5 \mu \mathrm{M}$ of dNTP and $7.8 \times 10^{3}$ copies of RNA template ( $\mathrm{n}=5$ for TFV-DP at 0,100 , and 1,250 $\mathrm{nM}, \mathrm{n}=10$ for TFV-DP at $300 \mathrm{nM}$, and $\mathrm{n}=14$ for TFV-DP at $600 \mathrm{nM}$ ). All fluorescence data were normalized between a baseline measurement at 360 seconds (after mixing was performed) as 0 , and maximum fluorescence at 1,050 seconds from the positive control (no TFV-DP added) as 1.

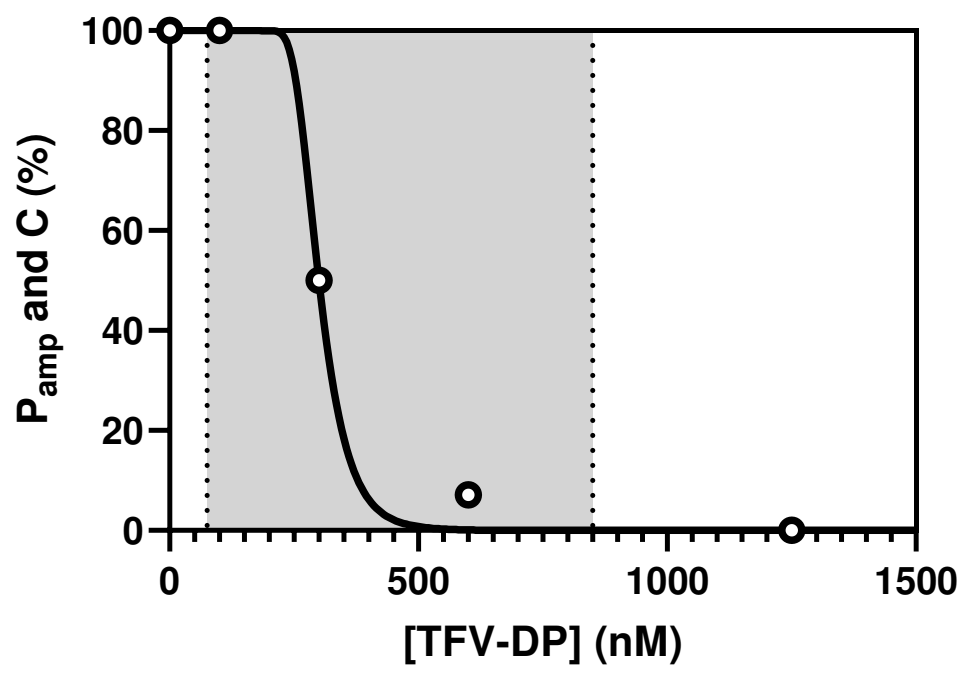

Figure 3. Analytical model (solid line) and experiments (circles) of the probability of amplification as a function of [TFV-DP] at $t=7.8 \times 10^{3}$ copies of RNA template and $s=21$ Uracil sites. Shaded area indicates the clinically relevant TFV-DP concentration range between $75 \mathrm{nM}$ (1 dose per week) and $850 \mathrm{nM}$ ( 7 dose per week). 




Figure 4. Probability of amplification as function of $R$, the ratio between dATP and TFV-DP concentration. The transition zone of the assay and the range of detection can be shifted by varying the components in the RT reaction mix number of sites, $s$, number of templates copies, $t$. Dashed lines indicated the conditions used in the current experiments and circles are experimental data.
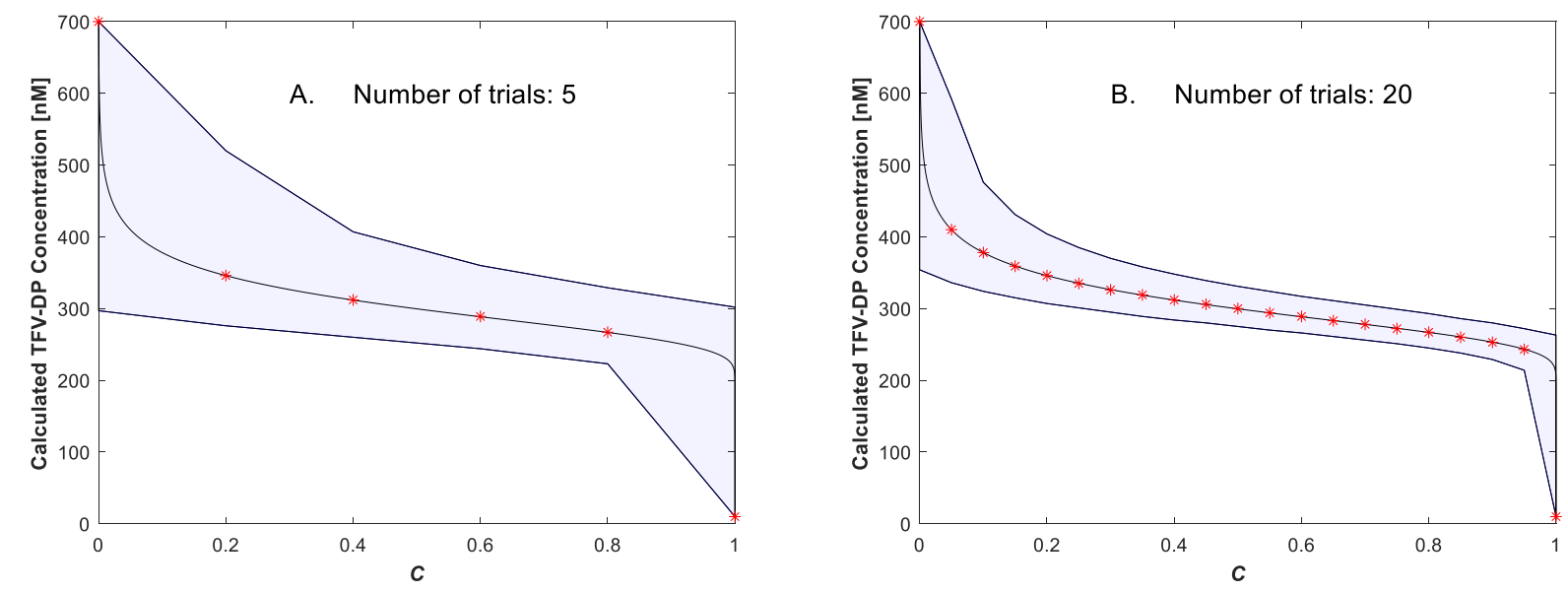

Figure 5. TFV-DP concentration as a function of the experimental positive trial proportion, $C$. Figure 5A and 5B represent the calculated TFV-DP for a pre-determined 5 and 20 repeated reactions, and a selected set of conditions of $t=7.8 \times 10^{3}$ copies of RNA template, $s=21$ Uracil sites, and $[d A T P]=0.375 \mu \mathrm{M}$. The line with the symbols represents the expected $[T F V-D P]$ given 
the experimental positive trial proportion. The shaded area represents the $95 \%$ confidence interval for each experimental outcome.

Table 1. Look up table of $p$-values for the hypothesis test that $[T F V-D P]>292 \mathrm{nM}$, as a function of the total number of trials, $N$, and the number of positive amplification reactions, $n$. Direct observation clinical studies show that patient samples with TFV-DP greater than $292 \mathrm{nM}$, or four doses per week, are adherent and protected from contracting HIV. Highlighted boxes in green represent $p<0.05$ or greater than $95 \%$ confidence that the hypothesis is true.

\begin{tabular}{|c|c|c|c|c|c|c|c|c|c|c|}
\hline & $\mathrm{N}=1$ & 2 & 3 & 4 & 5 & 6 & 7 & 8 & 9 & 10 \\
\hline $\mathrm{n}=0$ & 0.43 & 0.19 & 0.08 & 0.03 & 0.02 & 0.01 & 0.00 & 0.00 & 0.00 & 0.00 \\
\hline 1 & 1.00 & 0.68 & 0.40 & 0.22 & 0.11 & 0.06 & 0.03 & 0.01 & 0.01 & 0.00 \\
\hline 2 & & 1.00 & 0.82 & 0.58 & 0.37 & 0.23 & 0.13 & 0.07 & 0.04 & 0.02 \\
\hline 3 & & & 1.00 & 0.90 & 0.72 & 0.52 & 0.35 & 0.23 & 0.14 & 0.08 \\
\hline 4 & & & & 1.00 & 0.94 & 0.81 & 0.65 & 0.48 & 0.34 & 0.22 \\
\hline 5 & & & & & 1.00 & 0.97 & 0.88 & 0.75 & 0.60 & 0.45 \\
\hline 6 & & & & & & 1.00 & 0.98 & 0.92 & 0.82 & 0.69 \\
\hline 7 & & & & & & & 1.00 & 0.99 & 0.95 & 0.88 \\
\hline 8 & & & & & & & & 1.00 & 0.99 & 0.97 \\
\hline 9 & & & & & & & & & 1.00 & 1.00 \\
\hline 10 & & & & & & & & & & 1.00 \\
\hline
\end{tabular}




\section{References}

1. HIV/AID, WHO/HIV/2019; World Health Organization (WHO), 2019.

2. HIV/AIDS Statistics Overview 2019; Centers for Disesase Control and Prevention, 2019.

3. WHO Expands Recommendation on Oral Pre-Exposure Prophylaxis of HIV Infection (PrEP);

WHO/HIV/2015.48; World Health Organization (WHO), 2015.

4. HIV Basics; Centers for Disease Control and Prevention, 2019.

5. HIV Data and Trends U.S. Statistics; Centers for Disease Control and Prevention, 2020.

6. Youn, B., Shireman, T. I., Lee, Y., Galárraga, O. \& Wilson, I. B. Trends in medication adherence in HIV patients in the US, 2001 to 2012: an observational cohort study. J Int AIDS Soc 22, e25382 (2019).

7. Bukenya, D., Mayanja, B. N., Nakamanya, S., Muhumuza, R. \& Seeley, J. What causes nonadherence among some individuals on long term antiretroviral therapy? Experiences of individuals with poor viral suppression in Uganda. AIDS Research and Therapy 16, 2 (2019).

8. Kim, J., Lee, E., Park, B.-J., Bang, J. H. \& Lee, J. Y. Adherence to antiretroviral therapy and factors affecting low medication adherence among incident HIV-infected individuals during 2009-2016: A nationwide study. Sci Rep 8, 1-8 (2018).

9. Eaton, L. A. et al. Elevated HIV Prevalence and Correlates of PrEP Use Among a Community Sample of Black Men Who Have Sex With Men. JAIDS Journal of Acquired Immune Deficiency Syndromes 79, 339 (2018).

10. Pre-exposure prophylaxis (PrEP) for HIV prevention. Avert https://www.avert.org/professionals/hivprogramming/prevention/pre-exposure-prophylaxis (2016).

11. Hodes, R., Cluver, L., Toska, E. \& Vale, B. Pesky metrics: the challenges of measuring ART adherence among HIV-positive adolescents in South Africa. Critical Public Health 0, 1-12 (2018). 
12. While Adherence to ART Regimens Has Improved Among People With HIV, Rates Remain Suboptimal. AJMC https://www.ajmc.com/newsroom/while-adherence-to-art-regimens-hasimproved-among-people-with-hiv-rates-remain-suboptimal.

13. Castillo-Mancilla, J. R. \& Haberer, J. E. Adherence Measurements in HIV: New Advancements in Pharmacologic Methods and Real-Time Monitoring. Curr HIV/AIDS Rep 15, 49-59 (2018).

14. Abaasa, A. et al. Utility of Different Adherence Measures for PrEP: Patterns and Incremental Value. AIDS Behav 22, 1165-1173 (2018).

15. Rao, A., Tobin, K., Davey-Rothwell, M. \& Latkin, C. Social Desirability Bias and Prevalence of Sexual HIV Risk Behaviors Among People Who Use Drugs in Baltimore, Maryland: Implications for Identifying Individuals Prone to Underreporting Sexual Risk Behaviors. AIDS and behavior 21, (2017).

16. Haberer, J. E. et al. Real-Time Adherence Monitoring for HIV Antiretroviral Therapy. AIDS Behav 14, 1340-1346 (2010).

17. Garrison, L. E. \& Haberer, J. E. Technological methods to measure adherence to antiretroviral therapy and preexposure prophylaxis: Current Opinion in HIV and AIDS 12, 467-474 (2017).

18. Chai, P. R. et al. Utilizing an Ingestible Biosensor to Assess Real-Time Medication Adherence. J. Med. Toxicol. 11, 439-444 (2015).

19. Garofalo, R. et al. A randomized controlled trial of personalized text message reminders to promote medication adherence among HIV-positive adolescents and young adults. AIDS Behav 20, 10491059 (2016).

20. Sabin, L. L. et al. Improving Adherence to Antiretroviral Therapy With Triggered Real-time Text Message Reminders: The China Adherence Through Technology Study. JAIDS Journal of Acquired Immune Deficiency Syndromes 69, 551 (2015). 
21. Mayer, J. E. \& Fontelo, P. Meta-Analysis on the Effect of Text Message Reminders for HIV-Related Compliance. AIDS Care 29, 409-417 (2017).

22. Spratt, E. et al. Using Technology to Improve Adherence to HIV Medications in Transitional Age Youth: Research Reviewed, Methods Tried, Lessons Learned. J Gen Med (Dover) 1, (2017).

23. Haberer, J. Panel Discussion Innovative Measures of Adherence: New Thinking, New Approaches.

24. Günthard, H. F. et al. Antiretroviral Drugs for Treatment and Prevention of HIV Infection in Adults: 2016 Recommendations of the International Antiviral Society-USA Panel. JAMA 316, 191-210 (2016).

25. Pre-Exposure Prophylaxis (PrEP) | HIV Risk and Prevention | HIV/AIDS | CDC. https://www.cdc.gov/hiv/risk/prep/index.html (2019).

26. Antoniou, T., Park-Wyllie, L. Y. \& Tseng, A. L. Tenofovir: A Nucleotide Analog for The Management of Human Immunodeficiency Virus Infection. Pharmacotherapy: The Journal of Human Pharmacology and Drug Therapy 23, 29-43 (2003).

27. Tenofovir DF-Emtricitabine Truvada - Treatment - National HIV Curriculum. https://www.hiv.uw.edu/page/treatment/drugs/tenofovir-disoproxil-fumarate-emtricitabine.

28. Organization, W. H. \& UNAIDS. Antiretroviral medicines in low-and middle-income countries: forecasts of global and regional demand for 2013-2016. (World Health Organization, 2014).

29. Naswa, S. \& Marfatia, Y. S. Pre-exposure prophylaxis of HIV. Indian J Sex Transm Dis AIDS 32, $1-8(2011)$.

30. Nucleoside Reverse Transcriptase Inhibitor (NRTI) Definition. AIDSinfo https://aidsinfo.nih.gov/understanding-hiv-aids/glossary/902/nucleoside-reverse-transcriptaseinhibitor. 
31. Kearney, B. P., Flaherty, J. F. \& Shah, J. Tenofovir Disoproxil Fumarate. Clin Pharmacokinet 43, 595-612 (2004).

32. Castillo-Mancilla, J. R. et al. Tenofovir, Emtricitabine, and Tenofovir Diphosphate in Dried Blood Spots for Determining Recent and Cumulative Drug Exposure. AIDS Research and Human Retroviruses 121010062750004 (2012) doi:10.1089/aid.2012.0089.

33. Morrow, M. et al. Predictive Value of Tenofovir Diphosphate in Dried Blood Spots for Future Viremia in Persons Living With HIV. J Infect Dis 220, 635-642 (2019).

34. Castillo-Mancilla, J. R. et al. Tenofovir, Emtricitabine, and Tenofovir Diphosphate in Dried Blood Spots for Determining Recent and Cumulative Drug Exposure. AIDS Res Hum Retroviruses 29, 384-390 (2013).

35. Abaasa, A. et al. Utility of Different Adherence Measures for PrEP: Patterns and Incremental Value. AIDS Behav 22, 1165-1173 (2018).

36. Castillo-Mancilla, J. R. \& Haberer, J. E. Adherence Measurements in HIV: New Advancements in Pharmacologic Methods and Real-Time Monitoring. Curr HIV/AIDS Rep 15, 49-59 (2018).

37. Pratt, G. W., Fan, A., Melakeberhan, B. \& Klapperich, C. M. A competitive lateral flow assay for the detection of tenofovir. Analytica Chimica Acta 1017, 34-40 (2018).

38. Gandhi, M. et al. Development and Validation of an Immunoassay for Tenofovir in Urine as a RealTime Metric of Antiretroviral Adherence. EClinicalMedicine 2-3, 22-28 (2018).

39. Spinelli, M. A. et al. Low tenofovir level in urine by a novel immunoassay is associated with seroconversion in a preexposure prophylaxis demonstration project: AIDS 33, 867-872 (2019).

40. Koenig, H. C. et al. Urine assay for tenofovir to monitor adherence in real time to tenofovir disoproxil fumarate/emtricitabine as pre-exposure prophylaxis. HIV Medicine 18, 412-418 (2017). 
41. Gandhi, M. M. et al. Validation of a Urine Tenofovir Immunoassay for Adherence Monitoring to PrEP and ART and Establishing the Cutoff for a Point-of-Care Test. Journal of Acquired Immune Deficiency Syndromes 81, 72-77 (2019).

42. Anderson, P. L. What Can Urine Tell Us About Medication Adherence? EClinicalMedicine 2, 5-6 (2018).

43. Anderson, P. L. et al. Intracellular Tenofovir-Diphosphate and Emtricitabine-Triphosphate in Dried Blood Spots following Directly Observed Therapy. Antimicrob. Agents Chemother. 62, e01710-17 (2018).

44. Castillo-Mancilla, J. R. et al. Tenofovir Diphosphate in Dried Blood Spots Is Strongly Associated With Viral Suppression in Individuals With Human Immunodeficiency Virus Infections. Clin Infect Dis 68, 1335-1342 (2019).

45. Grant, R. M. et al. Uptake of pre-exposure prophylaxis, sexual practices, and HIV incidence in men and transgender women who have sex with men: a cohort study. The Lancet Infectious Diseases 14, 820-829 (2014).

46. Coyle, R. P. et al. Engagement in Mental Health Care is Associated with Higher Cumulative Drug Exposure and Adherence to Antiretroviral Therapy. AIDS Behav (2019) doi:10.1007/s10461-01902441-8.

47. Anderson, P. L. et al. Emtricitabine-Tenofovir Concentrations and Pre-Exposure Prophylaxis Efficacy in Men Who Have Sex with Men. Science Translational Medicine 4, 151ra125-151ra125 (2012).

48. An enzymatic assay to measure long-term adherence to pre-exposure prophylaxis and antiretroviral therapy | bioRxiv. https://www.biorxiv.org/content/10.1101/832410v1. 
49. Olanrewaju, A. O. et al. Enzymatic Assay for Rapid Measurement of Antiretroviral Drug Levels. ACS Sens. 5, 952-959 (2020).

50. Kokkula, C., Palanisamy, N., Ericstam, M. \& Lennerstrand, J. SYBR Green II Dye-Based RealTime Assay for Measuring Inhibitor Activity Against HIV-1 Reverse Transcriptase. Mol Biotechnol 58, 619-625 (2016).

51. Frezza, C., Balestrieri, E., Marino-Merlo, F., Mastino, A. \& Macchi, B. A novel, cell-free PCRbased assay for evaluating the inhibitory activity of antiretroviral compounds against HIV reverse transcriptase. J. Med. Virol. 86, 1-7 (2014).

52. Marino-Merlo, F. et al. Development and evaluation of a simple and effective RT-qPCR inhibitory assay for detection of the efficacy of compounds towards HIV reverse transcriptase. Appl. Microbiol. Biotechnol. 101, 8249-8258 (2017).

53. Lillis, L. et al. Factors influencing Recombinase Polymerase Amplification (RPA) assay outcomes at point of care. Mol Cell Probes 30, 74-78 (2016).

54. Piepenburg, O., Williams, C. H. \& Armes, N. A. Methods for multiplexing recombinase polymerase amplification. (2011).

55. Boyle, D. S. et al. Rapid detection of HIV-1 proviral DNA for early infant diagnosis using recombinase polymerase amplification. MBio 4, (2013).

56. Duwal, S., Sunkara, V. \& Kleist, M. von. Multiscale Systems-Pharmacology Pipeline to Assess the Prophylactic Efficacy of NRTIs Against HIV-1. CPT: Pharmacometrics \& Systems Pharmacology 5, 377-387 (2016).

57. Cantera, J. L. et al. Assessment of eight nucleic acid amplification technologies for potential use to detect infectious agents in low-resource settings. PLOS ONE 14, e0215756 (2019). 
58. TwistAmp manuals | Downloads | Support | TwistDx. https://www.twistdx.co.uk/en/support/manuals/twistamp-manuals.

66. PubChem [Internet]. Bethesda (MD): National Library of Medicine (US), National Center for Biotechnology Information; 2004-. PubChem Compound Summary for CID 5481180; [cited 2020 Nov. 9]. Available from: https://pubchem.ncbi.nlm.nih.gov/compound/tenofovir-diphosphate 67. PubChem [Internet]. Bethesda (MD): National Library of Medicine (US), National Center for Biotechnology Information; 2004-. PubChem Compound Summary for CID 15993, 2'Deoxyadenosine 5'-triphosphate; [cited 2020 Nov. 9]. Available from: https://pubchem.ncbi.nlm.nih.gov/compound/2_-Deoxyadenosine-5_-triphosphate 


\section{Figures}

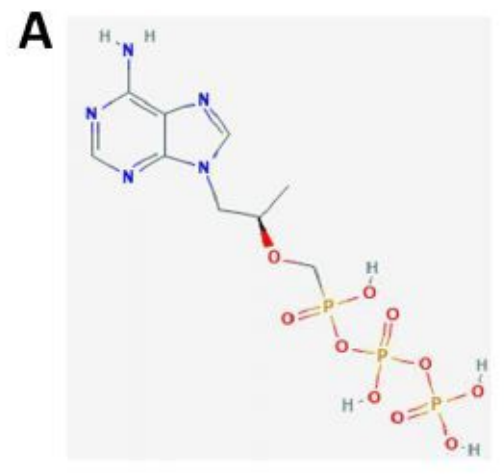

Tenofovir-Diphosphate (TFV-DP)

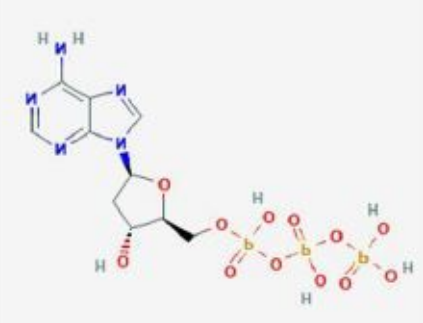

deoxyadenosine-Triphosphate

(dATP)
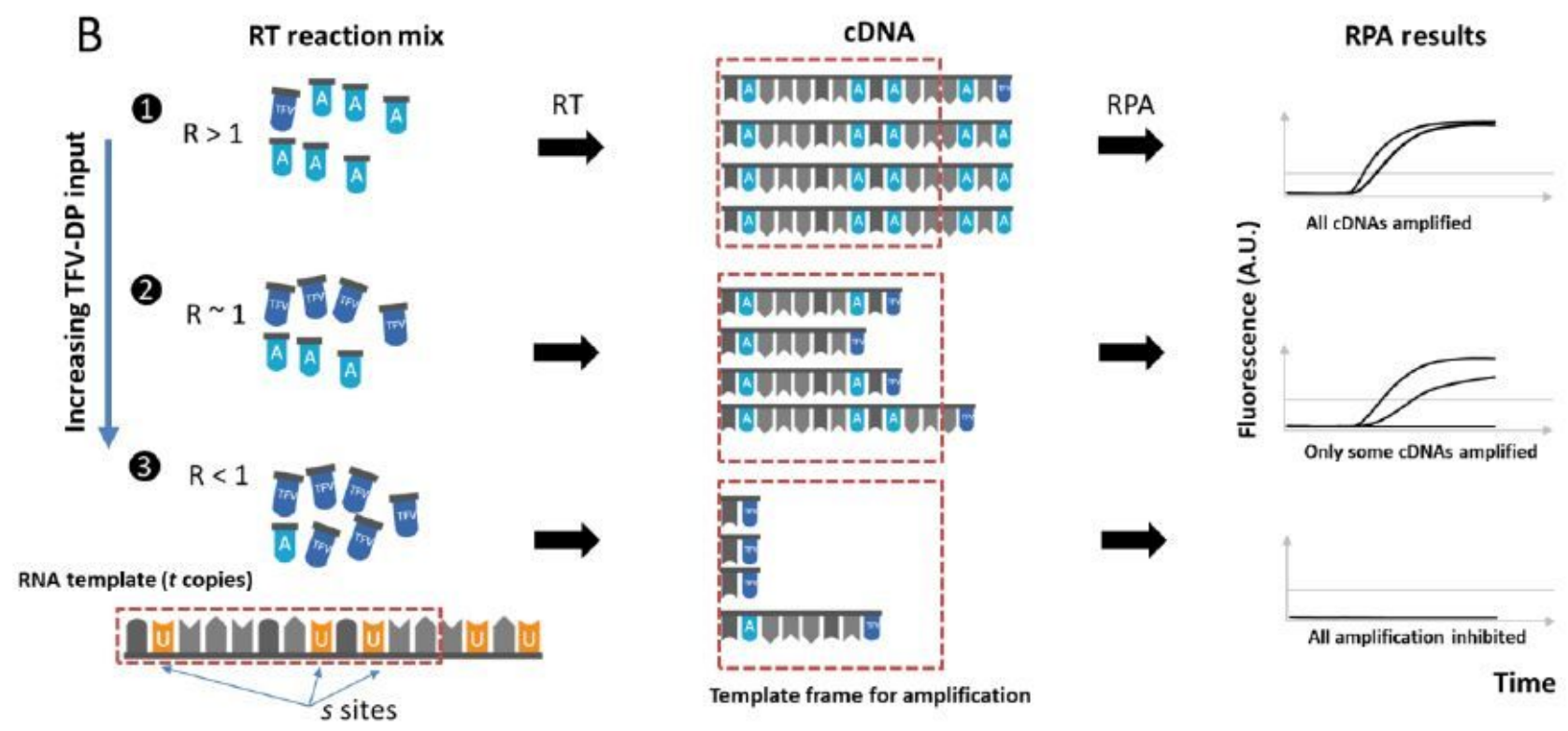

\section{Figure 1}

Overview of TFV-DP measurement using Reverse Transcription Isothermal Amplification Inhibition. (A) Molecular structural similarity between TFV-DP and dATP indicates that TFV-DP is a NRTI, blocking dATP binding to terminate HIV RT activities.59,60 (B). Modulation of the assay by competitive binding between dATP and TFV-DP during cDNA synthesis. The level of TFV-DP is determined by amplifying the RNA template that is reverse transcribed by RT. (1) If there is little or no TFV-DP present, the RT reversetranscribes the template RNA to form cDNA with a complete template frame for amplification, and the cDNA is amplified by RPA. (2) If there are moderate concentrations of TFV-DP compared to dATP, some of the CDNA chain may be terminated and thus only some full-length cDNA is formed. In this case only a fraction of amplification reactions will be positive. (3) When high concentrations of TFV-DP are present, nearly all cDNA is chain-terminated, resulting in RPA inhibition of all the samples. 

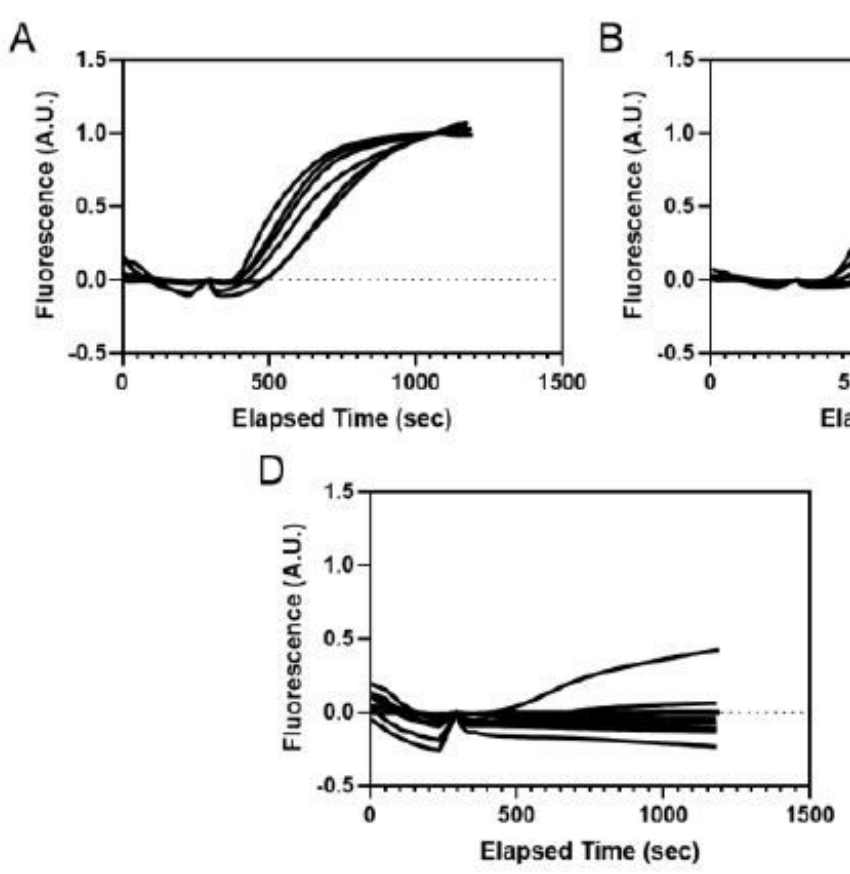
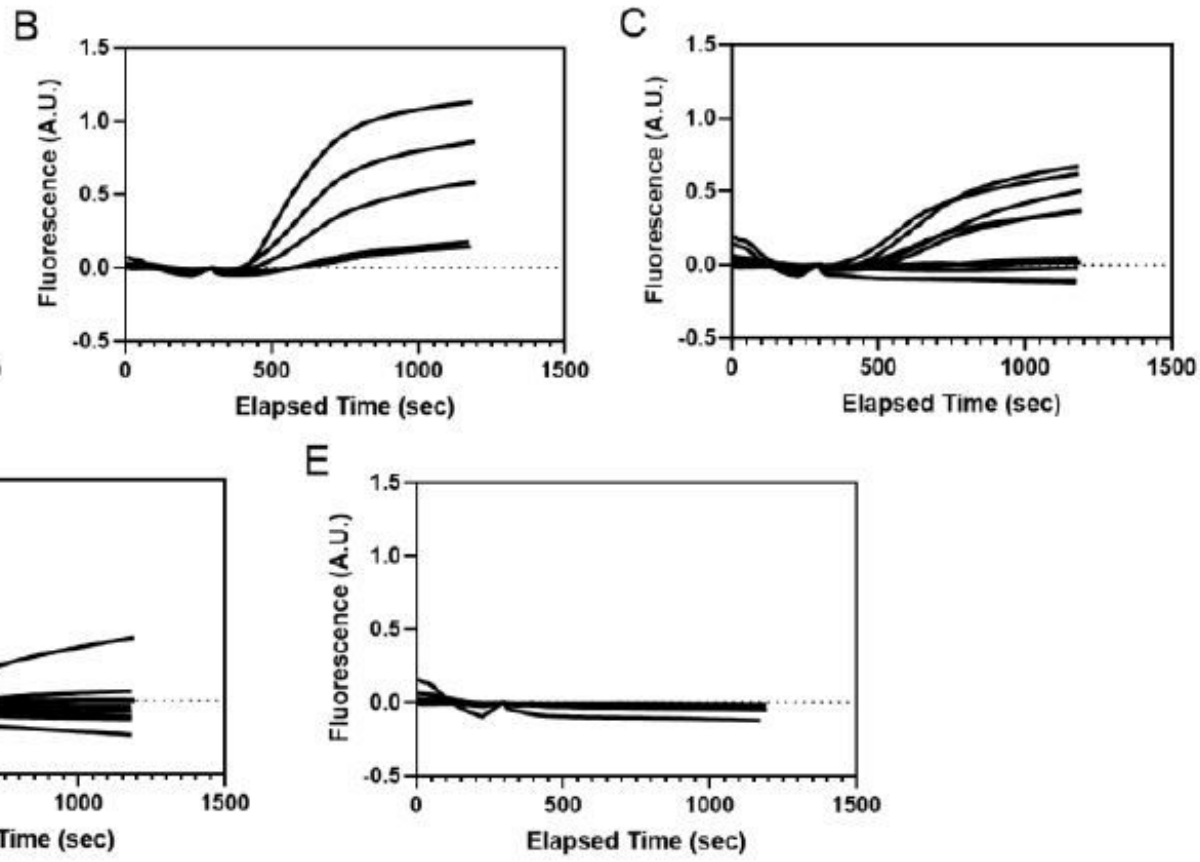

Figure 2

Real-time RPA fluorescence as a result of A) 0, B) $100 \mathrm{nM}$, C) $300 \mathrm{nM}$, D) $600 \mathrm{nM}$, and E) 1,250 nM TFVDP spiked into the RT reactions with $1.5 \mu \mathrm{M}$ of dNTP and $7.8 \times 103$ copies of RNA template ( $\mathrm{n}=5$ for TFVDP at 0,100 , and $1,250 \mathrm{nM}, \mathrm{n}=10$ for TFV-DP at $300 \mathrm{nM}$, and $n=14$ for TFV-DP at $600 \mathrm{nM}$ ). All fluorescence data were normalized between a baseline measurement at 360 seconds (after mixing was performed) as 0 , and maximum fluorescence at 1,050 seconds from the positive control (no TFV-DP added) as 1 . 


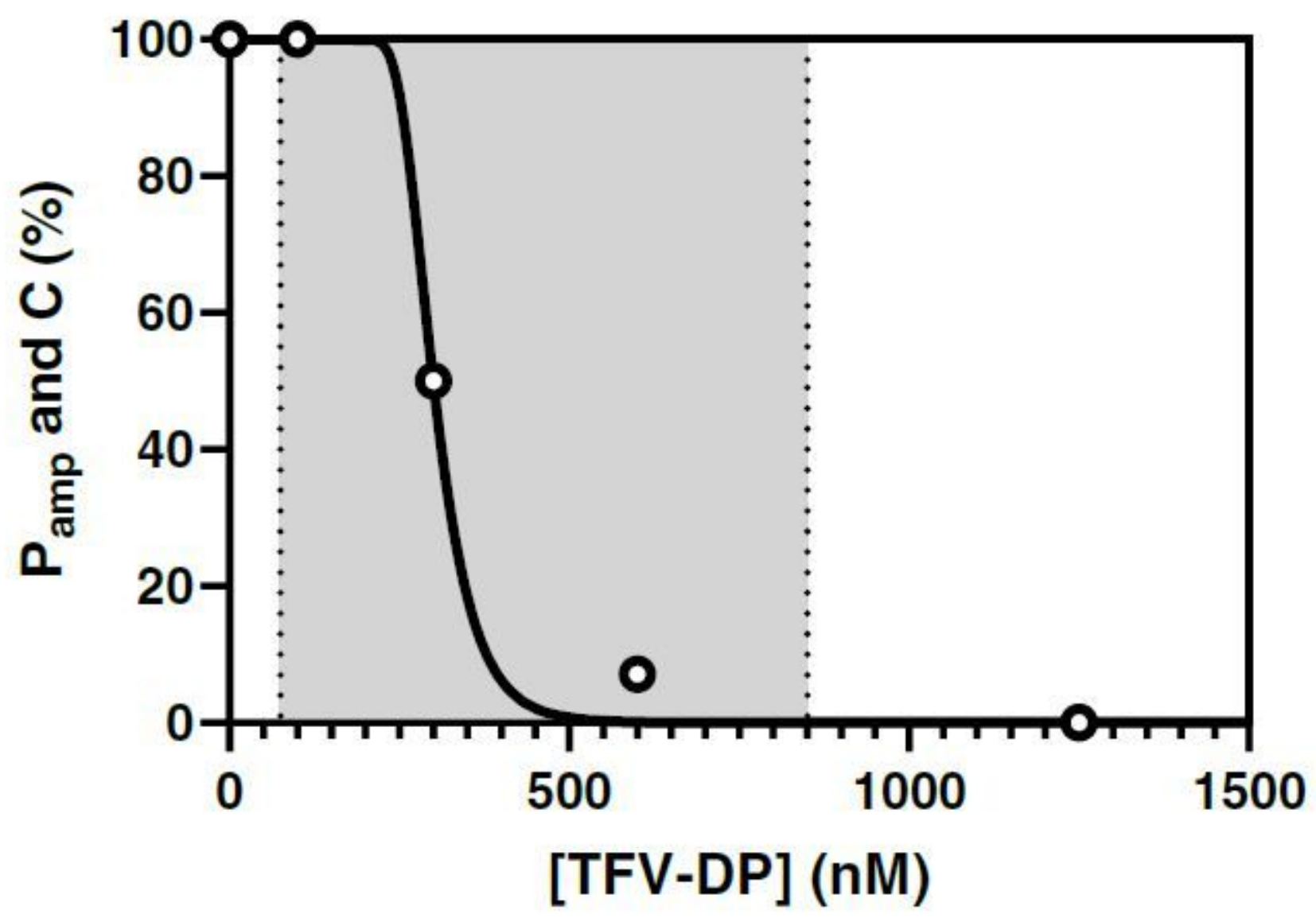

Figure 3

Analytical model (solid line) and experiments (circles) of the probability of amplification as a function of [TFV-DP] at $t=7.8 \times 103$ copies of RNA template and $s=21$ Uracil sites. Shaded area indicates the clinically relevant TFV-DP concentration range between $75 \mathrm{nM}$ (1 dose per week) and $850 \mathrm{nM}$ (7 dose per week). 


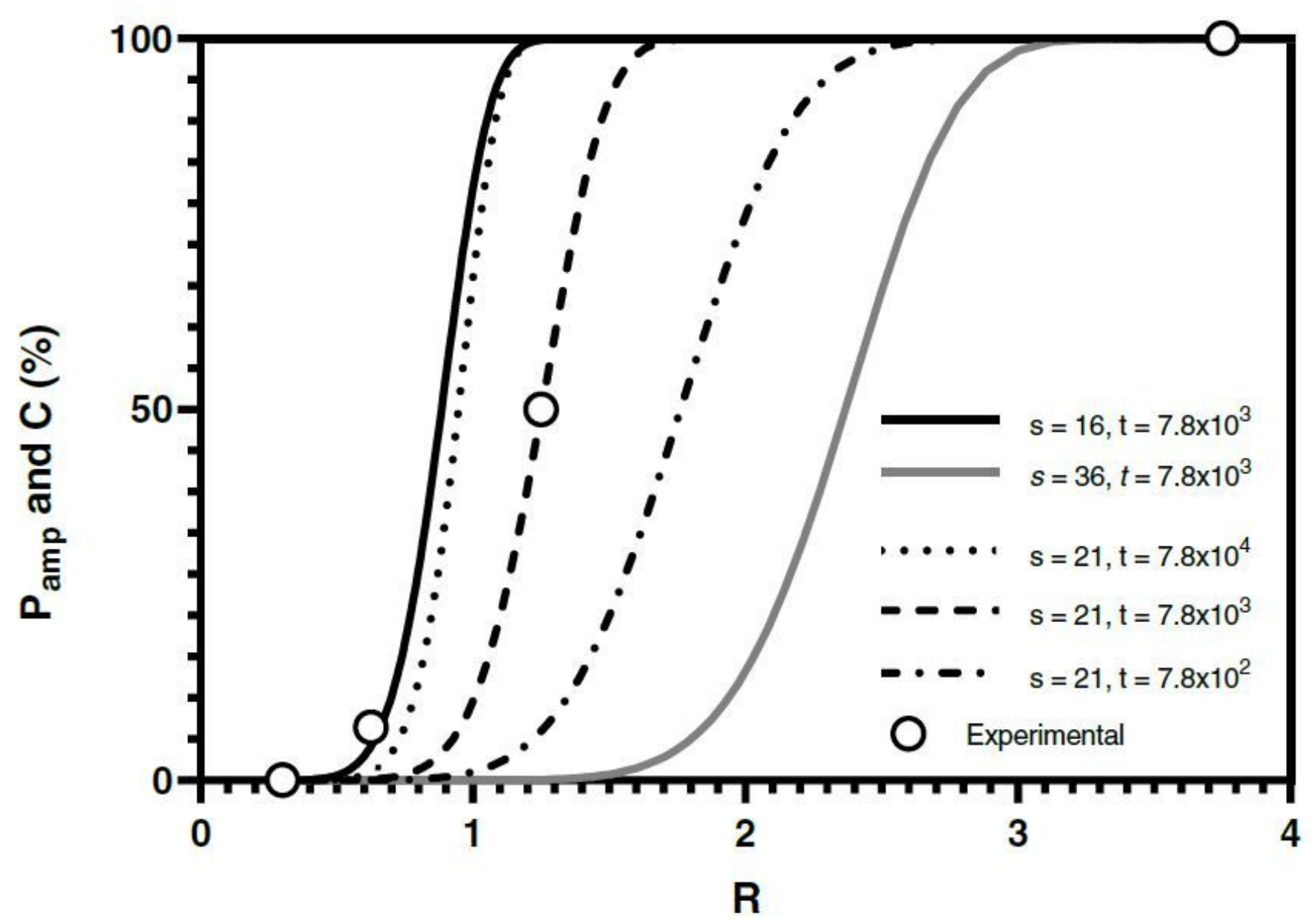

Figure 4

Probability of amplification as function of $R$, the ratio between dATP and TFV-DP concentration. The transition zone of the assay and the range of detection can be shifted by varying the components in the RT reaction mix number of sites, s, number of templates copies, t. Dashed lines indicated the conditions used in the current experiments and circles are experimental data. 

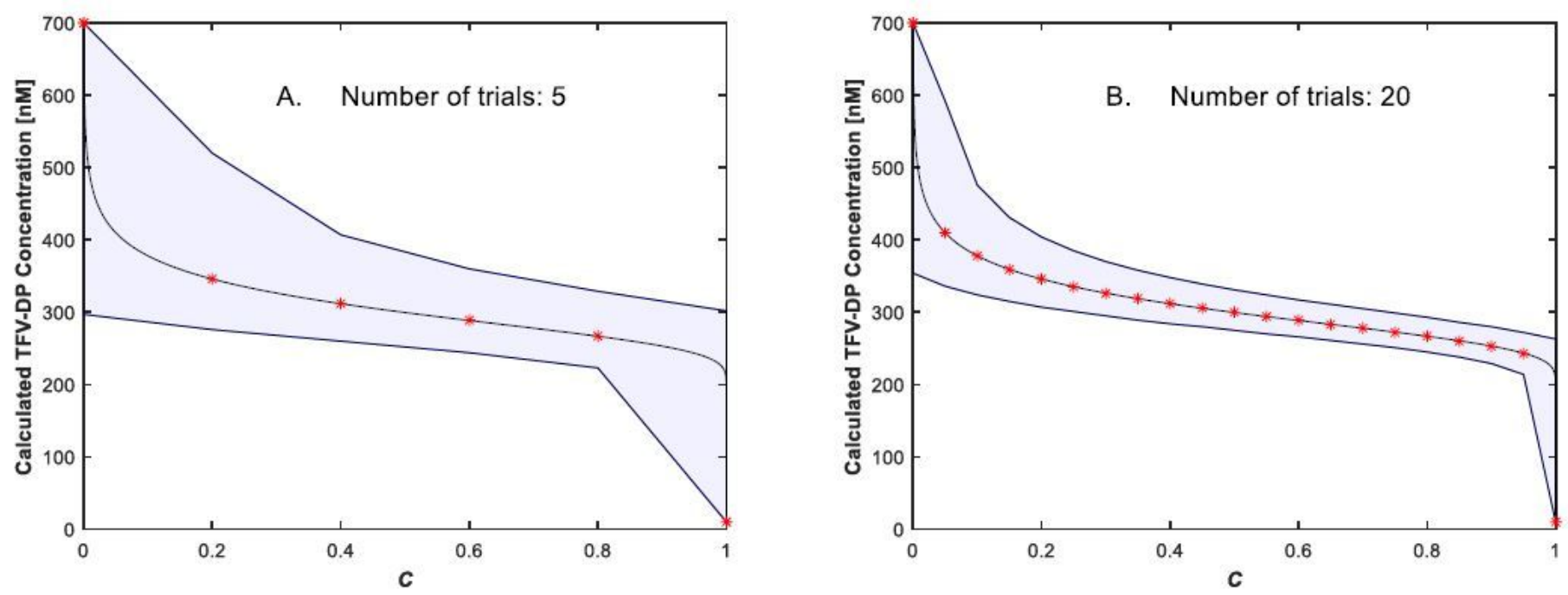

\section{Figure 5}

TFV-DP concentration as a function of the experimental positive trial proportion, C. Figure $5 \mathrm{~A}$ and $5 \mathrm{~B}$ represent the calculated TFV-DP for a pre-determined 5 and 20 repeated reactions, and a selected set of conditions of $t=7.8 \times 103$ copies of RNA template, $s=21$ Uracil sites, and [dATP] $=0.375 \mu \mathrm{M}$. The line with the symbols represents the expected [TFV-DP] given the experimental positive trial proportion. The shaded area represents the $95 \%$ confidence interval for each experimental outcome. 